\title{
The STING of metastasis
}

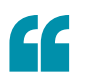

CIN has a

functional link to metastasis

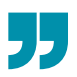

Simon Bradbrook/Macmillan Publishers Limited

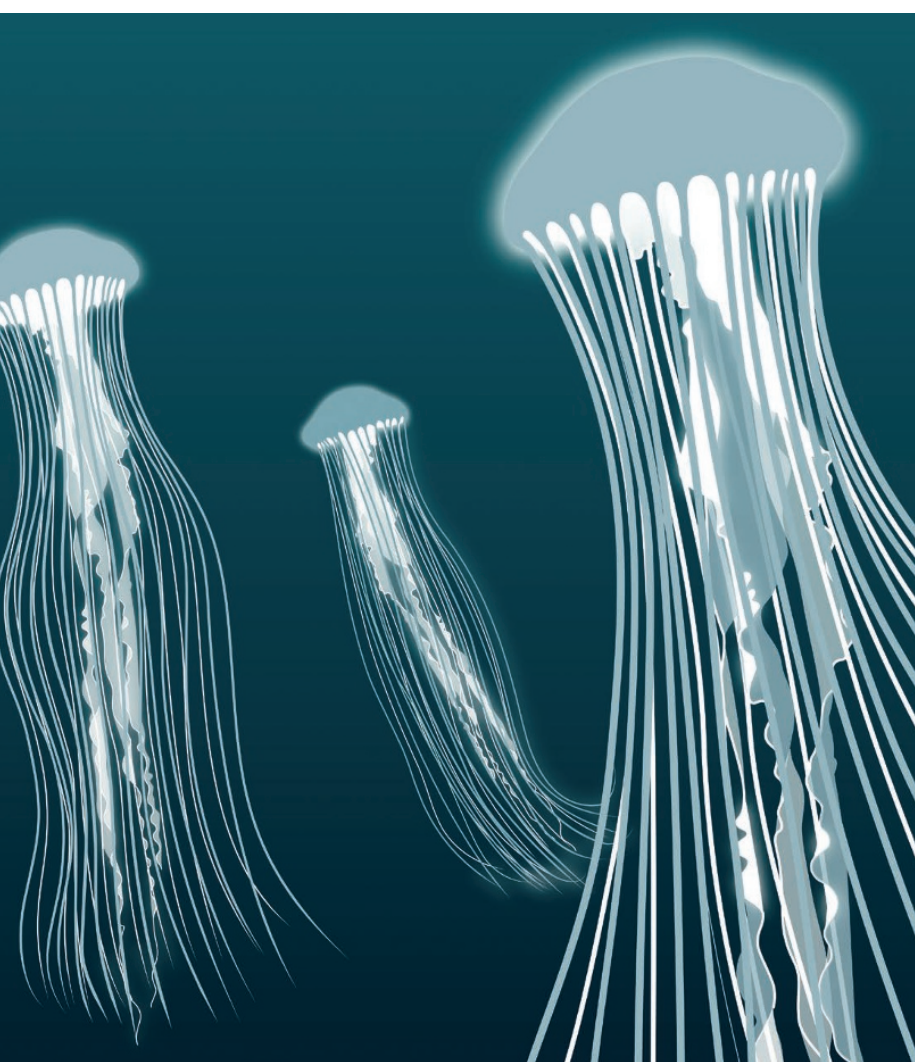

Although chromosomal instability (CIN) commonly occurs in cancers, whether it has a functional role in metastatic progression is unclear. Bakhoum, Ngo et al. suggest that CIN can promote metastasis through a cyclic GMP-AMP synthase (cGAS)-stimulator of interferon genes (STING) pathway-dependent response to cytosolic DNA.

Several lines of evidence indicated that CIN is associated with metastasis in various human tumour types. To investigate causality, the authors transplanted metastatic cancer cell lines (MDA-MB-231 human breast cancer, 4T1 mouse breast cancer and H2030 human lung cancer cells) in which CIN was suppressed into mice.
To suppress CIN, the kinesin-like proteins KIF2B or KIF2C were overexpressed in the cells - such cells retain their aneuploid karyotypes during proliferation but otherwise have chromosomal stability. Intracardiac injection of MDA-MB-231 cells or H2030 cells overexpressing KIF2B or KIF2C (CIN-low cells) revealed that these cells are less able to colonize metastatic sites compared with cells overexpressing a control kinesin-like protein (KIF2A) that did not affect CIN (CIN-high cells). This translated to increased survival for the mice injected with CIN-low cells. The role of CIN in spontaneous metastasis was then examined. Tumours formed from the injection of CIN-high or CIN-low MDA-MB-231 or 4T1 cells in the mammary fat pad of mice were surgically resected and subsequent metastases were analysed. Suppression of CIN did not reduce the efficacy of primary tumour implantation but did reduce metastasis and prolong survival of the mice.

Analysis of the cells that metastasized indicated that regardless of the CIN status of the initial injected cells, metastases were enriched for cells with high levels of CIN. Furthermore, RNA sequencing (RNA-seq) of bulk and single MDA-MB-231 lines showed that CIN-high cells were enriched not only for expression of metastasis-related genes but also genes involved in epithelial-mesenchymal transition (EMT), suggesting that EMT could be involved in metastasis of CIN-high cells.

Single-cell RNA-seq also indicated a correlation of inflammationrelated gene expression, similar to that induced during the sensing of cytosolic viral DNA, with CIN and EMT. Genomic DNA can be released into the cytosol through rupture of micronuclei, and the authors showed that CIN-high or metastatic cells contained more micronuclei and higher levels of cytosolic DNA than CIN-low or primary tumour-derived cells. This effect could be reduced by suppression of micronuclear envelope rupture. Further data indicated that chromosome missegregation can result in the presence of chromosome-derived DNA in the cytosol.

Double-stranded cytosolic DNA is typically sensed by the cGAS-STING pathway, which activates interferon and canonical nuclear factor- $\kappa \mathrm{B}$ (NF- $\kappa \mathrm{B})$ signalling. Although the localization of cGAS at micronuclei and levels of STING in CIN-high cells was consistent with increased pathway activation, there was no evidence of type I interferon or canonical $\mathrm{NF}-\kappa \mathrm{B}$ activation. However, the non-canonical NF- $\kappa$ B pathway was active in CIN-high cells, and STING depletion reduced its activation. There was also a correlation between CIN-high and non-canonical NF- $\kappa \mathrm{B}$ gene activation in the authors' RNAseq data as well as publically available RNA-seq data from breast tumours. Finally, MDA-MB-231 CIN-high cells depleted of STING or non-canonical NF- $\kappa$ B pathway members had a reduced ability to colonize metastatic sites following intracardiac injection compared with controls.

Overall, these data indicate that CIN has a functional link to metastasis and might help explain previous observations that CIN and aneuploidy can have tumour-suppressive effects in early tumorigenesis.

Sarah Seton-Rogers

ORIGINAL ARTICLE Bakhoum, S. F., Ngo, B. et al. Chromosomal instability drives metastasis through a cytosolic DNA response. Nature 553 , 467-472 (2018) 